\title{
A PROTECTED DATASHARING FOR COLLISIONSECUREDYNAMIC CLOUD
}

\author{
Kiran Kumar Wali ${ }^{1}$, Bindu Madhavi ${ }^{2}$, M. Nagendra ${ }^{3}$ \\ ${ }^{1}$ Student, Department of Computer Science ,T JIT , Karnataka, India \\ ${ }^{2}$ Associate professor Department of Computer Science ,T JIT , Karnataka, India \\ ${ }^{3}$ Associate professor Department of Computer Science, S.K.University. Karnataka, India
}

\begin{abstract}
Profited from distributed computing, clients can accomplish a successful and practical methodology for information sharing among gathering individuals in the cloud with the characters of low upkeep and little administration cost. In the interim, we should give security sureties to the sharing information documents since they are outsourced. Shockingly, on account of the regular change of the participation, sharing information while giving security safeguarding is still a testing issue, particularly for an untrusted cloud because of the plot assault. In addition, for existing plans, the security of key dispersion depends on the safe correspondence channel in any case, to have such channel is a solid supposition and is troublesome for practice. In this paper, we propose a safe information sharing plan for element individuals. Firstly, we propose a protected path for key conveyance with no safe correspondence channels, and the clients can safely get their private keys from gathering chief Secondly, our plan can accomplish fine-grained access control, any client in the gathering can Outilize the source in the cloud and disavowed clients can't get to the cloud again after they are denied. Thirdly, we can shield the plan from conspiracy assault, which implies that denied clients can't get the first information record regardless of the possibility that they plot with the untrusted cloud. In our methodology, by utilizing polynomial capacity, we can accomplish a safe client denial plan. At long last, our plan can accomplish fine productivity, which implies past clients need not to overhaul their private keys for the circumstance either another client joins in the gathering or a client is disavowe from the gathering.
\end{abstract}

Keywords: Access Control, Privacy-Preserving, Key Distribution, Cloud Computing. $* * *$

\section{INTRODUCTION}

Distributed computing, with the characteristics of regular data sharing and low upkeep, gives a prevalent use of advantages. In circulated figuring, cloud organization suppliers offer an impression of boundless storage space for clients to host data [1]. It can offer clients some help with relocating in order to diminish their budgetary overhead of data organizations the close-by organizations system into cloud servers. In any case, security concern transform into the key restriction as we now outsource the limit of data, which is possibly sensitive ,to cloud suppliers. To ensure data security, an average philosophy is to encode data records before the clients exchange the mixed data into the cloud [2]. Sadly, it is difficult to plot a sheltered and beneficial data sharing arrangement, especially for dynamic social affairs in the cloud.

we design a secure anti-collusion data sharing scheme for dynamic groups in the cloud. In our scheme, the userscan securely obtain their private keys from group manager Certificate Authorities and secure communication channels. Also, our scheme is able to support dynamic groups efficiently,

when a new user joins in the group or a user is revoked from the group, the private key of the other users do not need to be recomputed and updated.

\section{Problem Statement}

To cloud providers. To preserve data privacy, a common approach is to encrypt data files before the clients upload the encrypted data into the cloud [2]. Unfortunately, it is difficult to design a secure and efficient data sharing scheme, especiallyfo dynamic groups in the cloud.

\section{RELATED WORK}

In this paper, we have a tendency to propose a secure information sharing set up, which might accomplish secure key appropriation and information sharing for part bunch. The first commitments of this set up include: one.We provides a safe approach to dispersion key with no secure correlation channels. The clients will safely acquire their private keys from gathering director with no Certificate Authorities due to the check for individuals normally key of the consumer. 2. This arrange will evoke finegrained access control, with the help of the gathering consumer list, any consumer within the gathering will utilize the source within the cloud and disclaim purchasers cannot get to the cloud once more once they're renounced. 3. We suggest a secure info sharing arrange which might be shielded from plot attack. The unacknowledged purchasers can't have the capability to induce the primary info documents once they're denied in spite of of the very fact that they arrange with the untrusted cloud. Our arrange can do secure consumer 
resignation with the help of

polynomial capability. 4.The proposed arrange will support dynamic gatherings effectively, when another consumer joins within the gathering or a consumer is disavowed from the gathering, the non-public keys of alternate purchasers do not ought to be recomputed and upgraded. 5. Security examination to demonstrate the protection of our arrange. In extension, we tend to in addition perform reenactments to exhibit the flexibility of our arrange.

\section{EXISTING SYSTEMS}

-Kallahalla et alpresented a cryptographic stockpiling structure that enables secure data sharing on untrustworthyservers considering the strategies that parceling records into filegroups and scrambling each filegroup with an archive blockkey.

- $\mathrm{Yu}$ et alexploited and joined methodologies of key methodology quality based encryption, delegate reencryption and impassive re-encryption to achieve finegrained data access control without uncovering data substance.

\section{Disadvantage of Existing System}

- The record piece keys ought to be redesigned and circled for a customer revocation thusly, the structure had a significant key appointment overhead.

- The complexities of customer participation and repudiation in these arrangements are straightly extending with the amount of data proprietors and the denied customers.

- The single-proprietor way may disturb the execution of employments, where any part in the social affair can use the cloud organization to store and bestow data records to others.

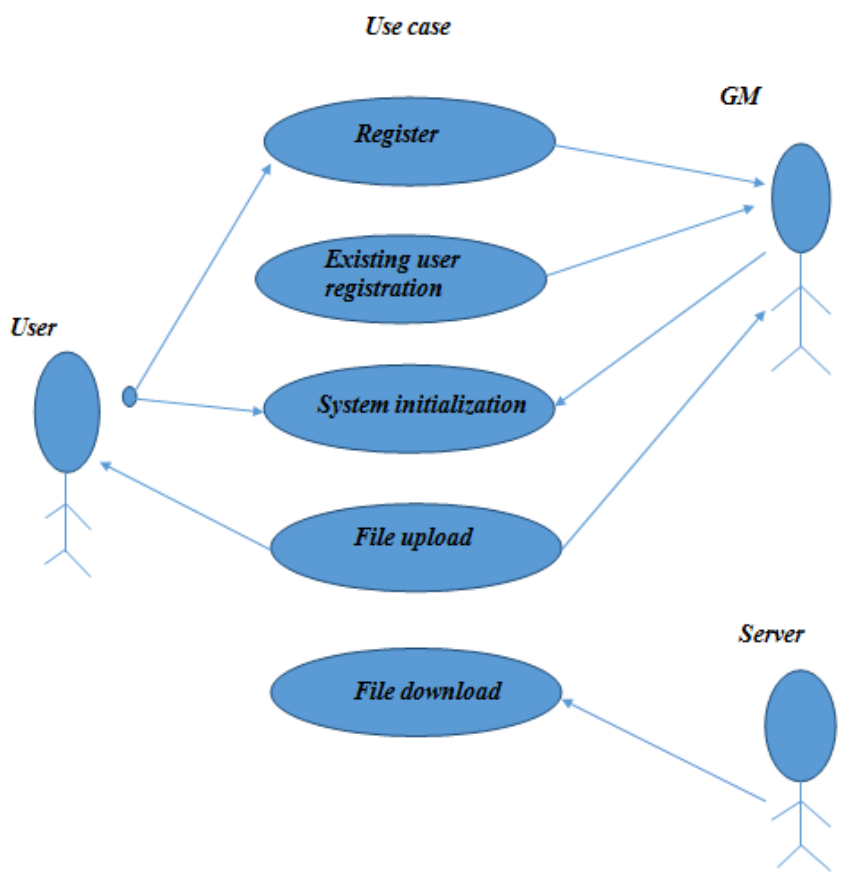

\section{PROPOSED SYSTEM}

In this paper, we propose a sheltered data sharing arrangement, which can finish secure key flow and data sharing for component bundle. We give a protected way to deal with key allocation with no sheltered correspondence channels. The customers can securely obtain their private keys from get-together boss with no Certificate Authorities on account of the check for general society key of the customer. Our arrangement can perform fine-grained access control, with the help of the social event customer list, any customer in the get- together can use the source in the cloud and revoked customers can't get to the cloud again after they are repudiated. We propose a protected data sharing arrangement which can be protected from plot attack. The disavowed customers can not have the ability to get th main data reports once they are renounced paying little mind to the likelihood that they arrange with the untrusted cloud. Our arrangementcan finish secur customer renouncemnt with the help of polynomial limit. Our arrangement can support dynamic social occasions capably, when another customer joins in the get-together or a customer is renounced from the get-together, the private keys of substitute customers don't tought to be recomputed and redesigned. We give security examination to exhibit the security of our arrangement.

\section{Advantages of proposed system}

- The estimation cost is unessential to the amount of denied customers in RBAC arrangement. The reason is that paying little respect to what number of customers are denied, the operations for people to unscramble the data records almost proceed as some time recently.

- The cost is unimportant to the amount of the repudiated customers. The reason is that the figuring cost of the cloud for report move in our arrangement includes two affirmations for imprint, which is immaterial to the amount of the repudiated customers. The clarification behind the little figuring cost of the cloud in the time of archive move in RBAC arrangement is that the affirmations between correspondence substances are not stressed in this arrangement.

- In our arrangement, the customer channel safel procure their private keys from social occasion

head Certificate Authorities and secure correspondenc e channels. Similarly, our arrangement can reinforce dynamic social affairs adequately, when another customer joins in the get-together or a customer is repudiated from the get-together, the private keys of interchange customers don't thought to be recomputed and redesigned. 


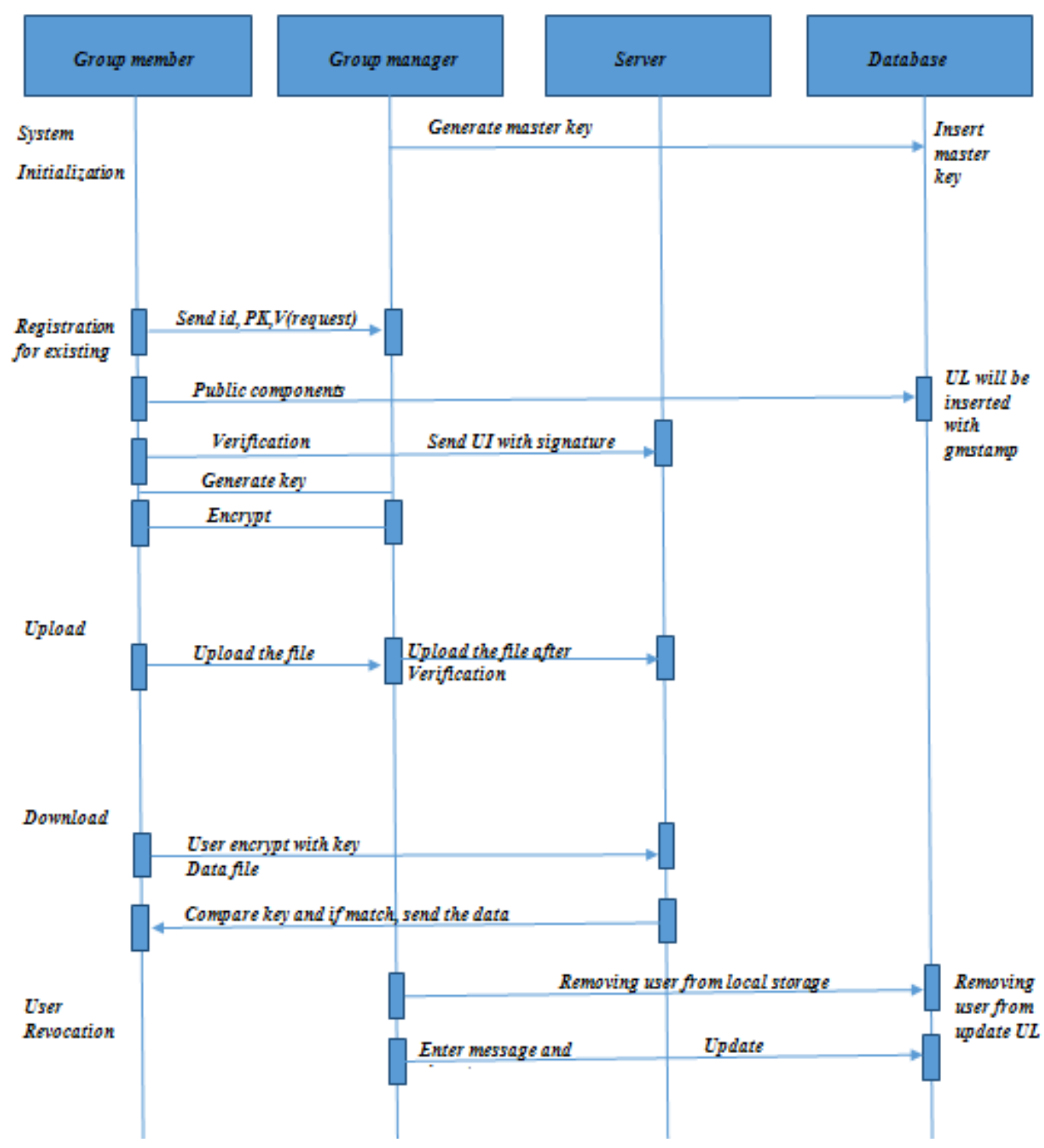

\subsection{SYSTEM DESIGN}

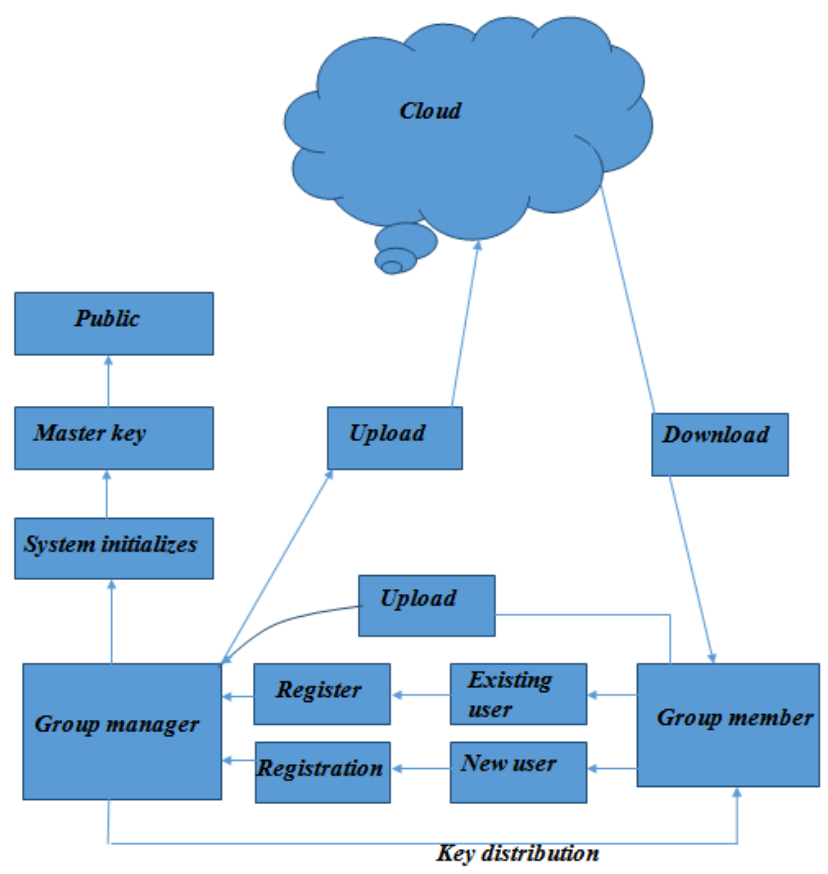

\section{System Initialization}

The group manager takes charge of this operation. He generates a bilinear map group system then selects two random elements and a number then computes and At last, the group managerpublishes the parameters where is hash function: is hash function: and is a symmetric encryption algorithm. Besides, the group manager will keep the parameters as the secret master key.

\section{Registrationfor Existing User}

This operation is performed by user the group manager .First of all, the user sends as a request to the group manager, the identity of the user, is the publi key used in the symmetric encryption algorithm, the account user used to pay for the registration, which is related to the identity of the user, and is a random number selected by the user.

File Upload

File Upload is done by the users in the group.Encrypt the file and outsource to the cloud.Encryption key would be generated once the file is encrypted and outsourced.users in the same group can upload and encrypt the file. 


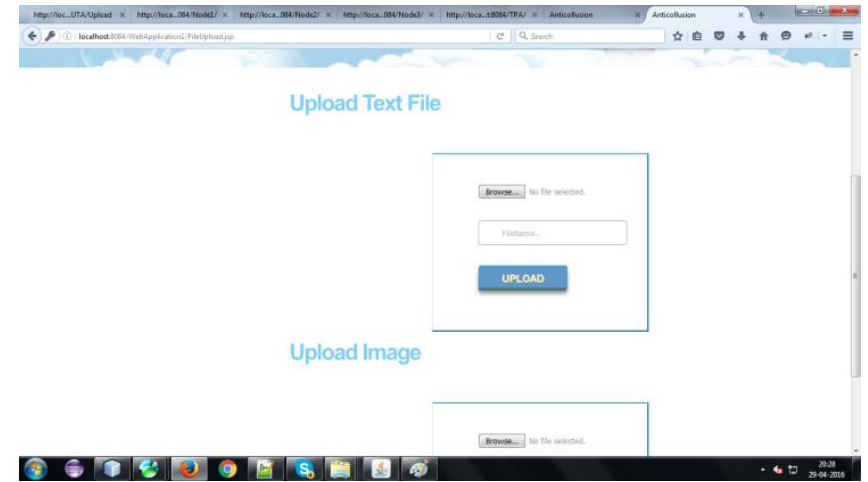

File Download

Download the file usiong the symmetric key and download the file..Users in the same group can download the file.

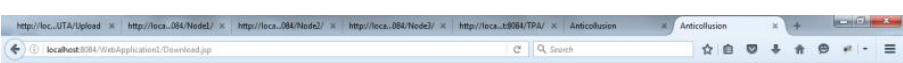

Anti Collution su vou tem somen nart

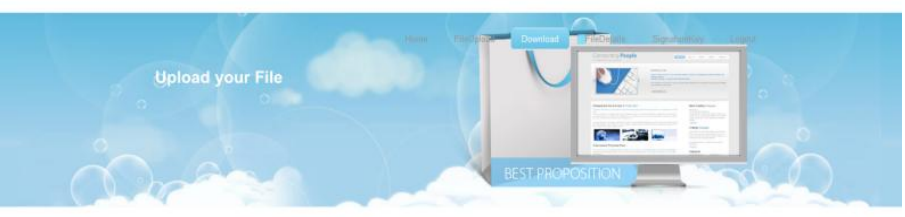

Download

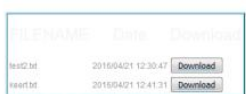

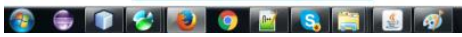

Account Revoke
Account revocation is done by the Group manager.If the user is found adversary .Once the user is revoked from the group, user cannot perform any action fpor the same group.

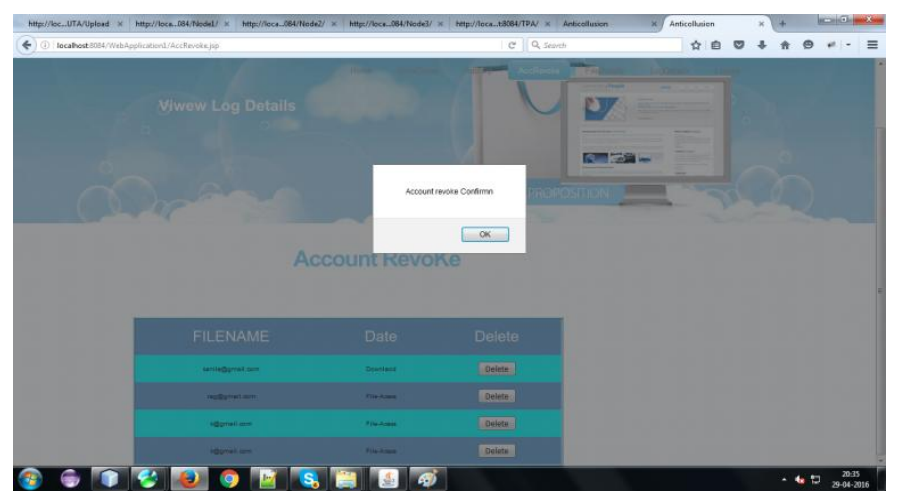

Diffie HellmanAlgorithm:

STEP 1: Alice and Bob agree to use Modulus

$\mathrm{P}=67$ \& base $\mathrm{g}=8$

STEP 2: Alice choose a Secret Integer a=6, Then ends Bob $\mathrm{A}=\mathrm{g}^{\mathrm{a}} \operatorname{modp}$

$\mathrm{A}=8^{9} \bmod 45=9$

STEP3: Bob Chooses a Secret Integer $b=15$, Then sends Alice $\mathrm{B}=\mathrm{g}^{\mathrm{b}} \bmod \mathrm{p}$

$\mathrm{B}=8^{17} \bmod 45=21$

STEP4: Alice Computes $S=B^{a}$ modp

$\mathrm{S}=12^{7} \bmod 45=5$

STEP5: Bob Computes $S=A^{b} \bmod p$

$\mathrm{S}=5^{13}$ Mod 45=6

STEP6: Alice \& Bob Shared a Secret e they will Mutually Authenicate \& they can share a Secret no(2)

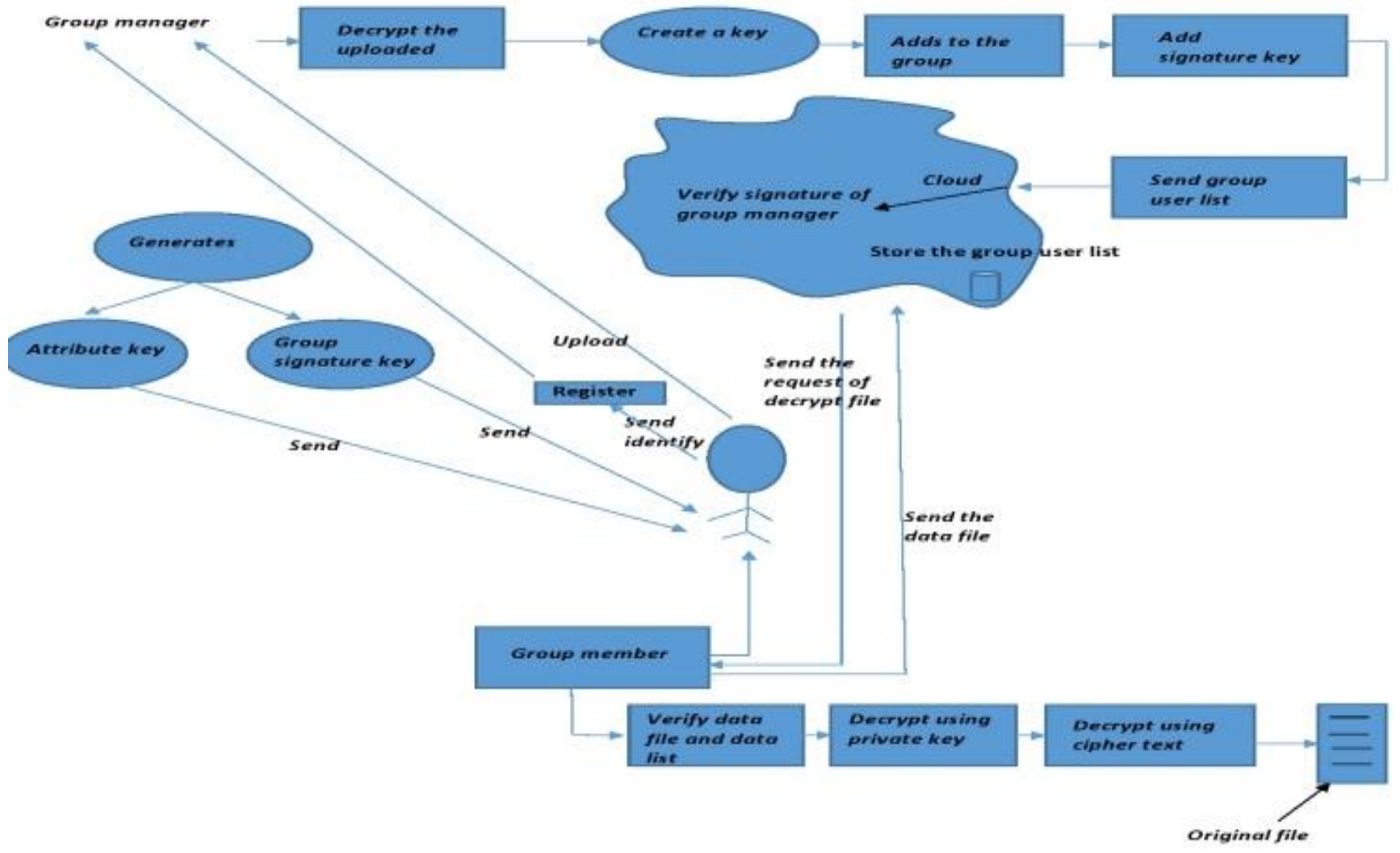




\section{RESULTS}

\section{Group Addition}

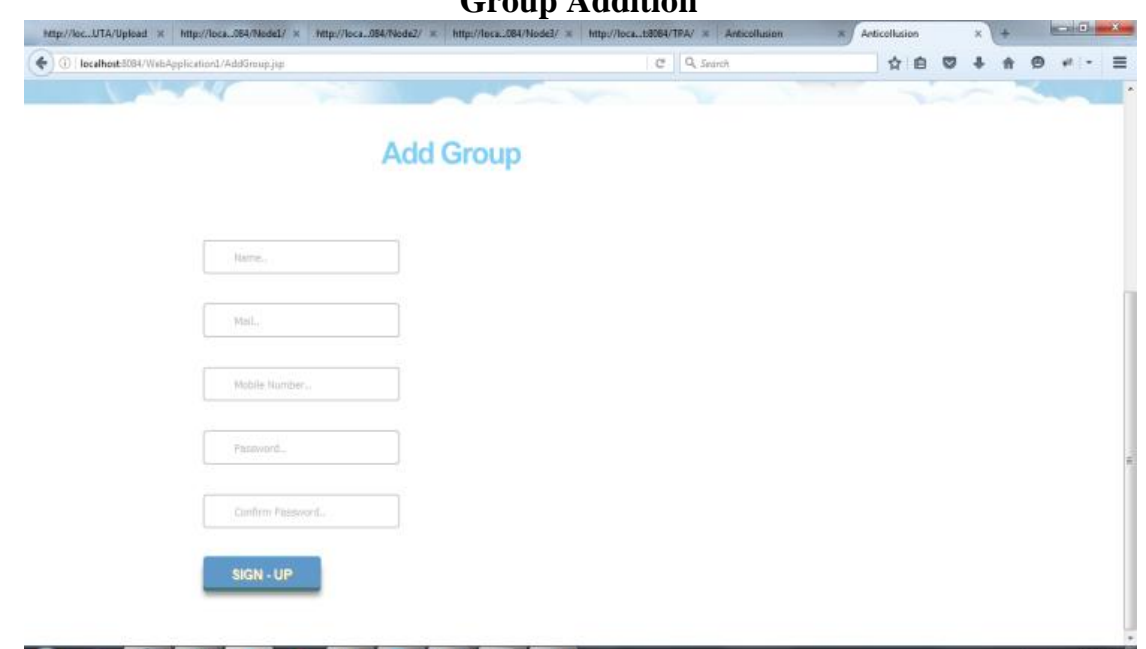

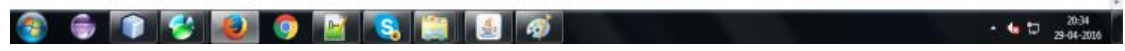

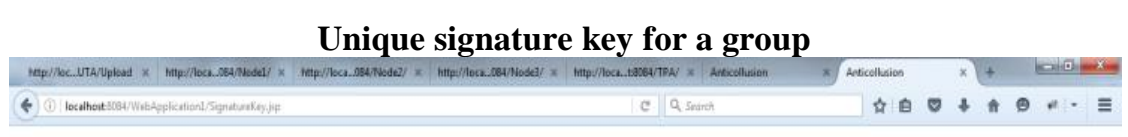

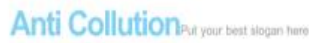

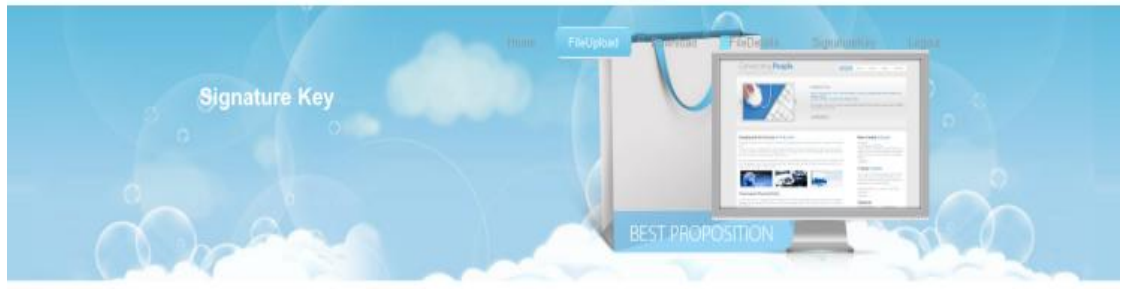

Signature Key

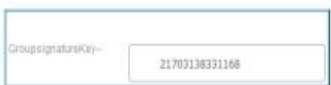

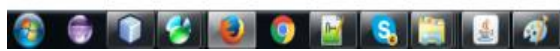

The group user can view files with group generation key

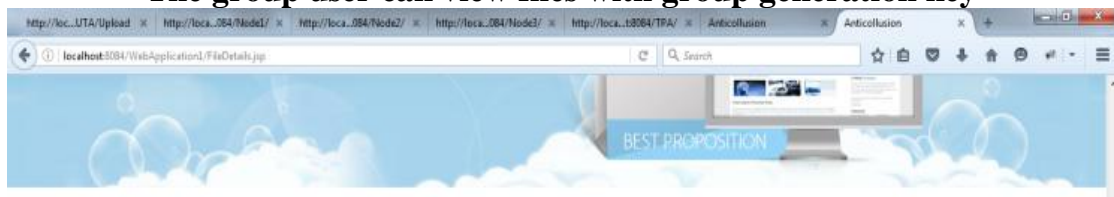

File Details

\begin{tabular}{|c|c|c|c|c|}
\hline FILENAME & Date & Delete & View & \\
\hline wat & masost t2men & Delets & & Vow \\
\hline$m$ & $2418029+12+12$ & Deektet & & Vew \\
\hline
\end{tabular}

Image Details

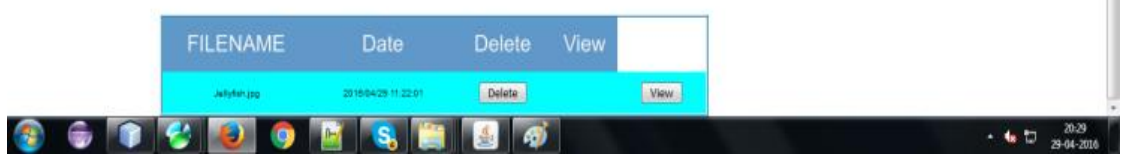




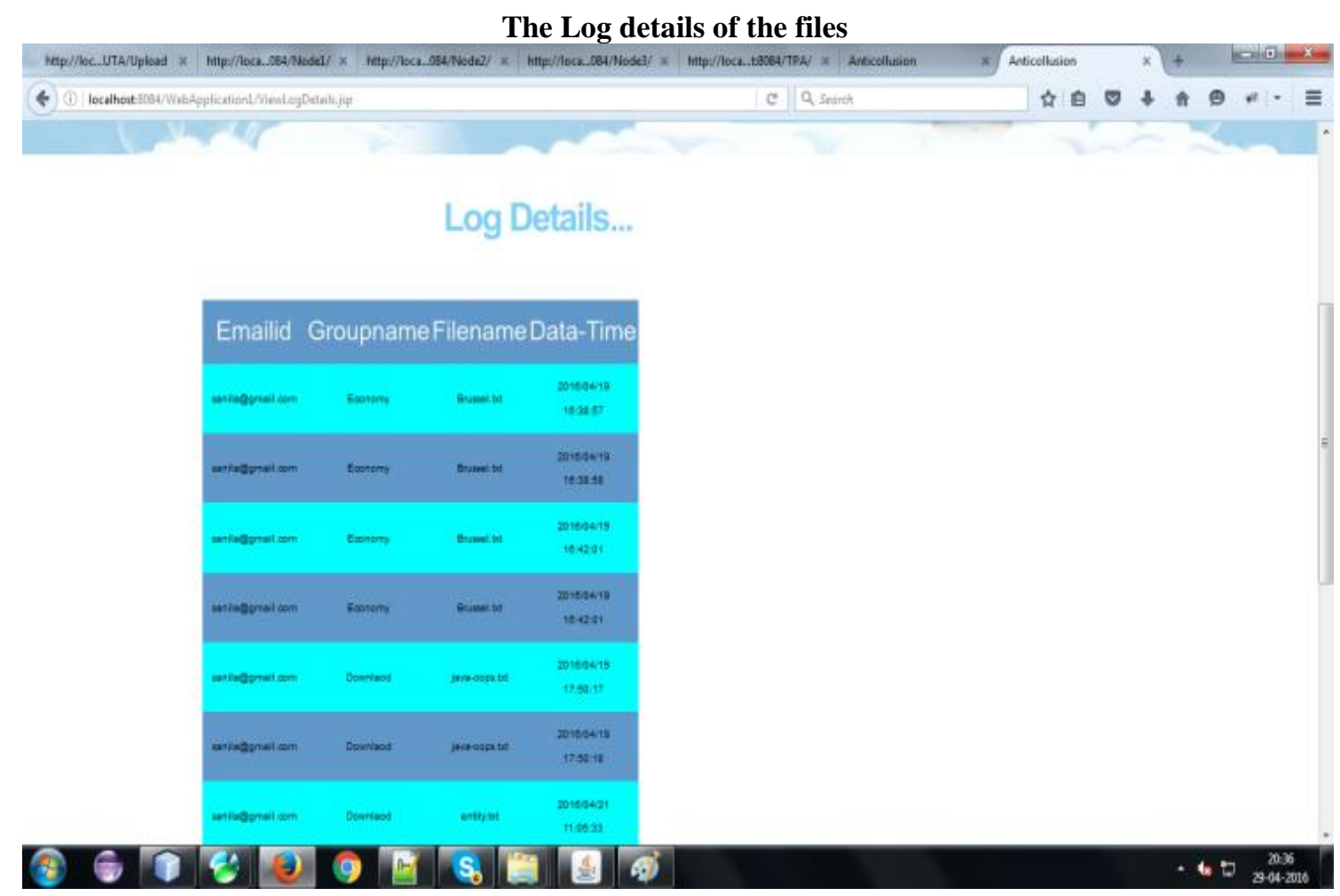

\section{CONCLUSIONS}

In this paper, we outline a protected against conspiracy information sharing plan for element bunches in the cloud. In our plan, the clients can safely get their private keys from gathering administrator Certificate Authorities and secure correspondence channels. Our plan can bolster dynamic gatherings productively, when another client joins in the gathering or a client is disavowed from the gathering, the private keys of alternate clients don't should be recomputed and overhauled. Besides, our plan can accomplish secure client repudiation, the denied clients can not have the capacity to get the first information documents once they are renounced regardless of the possibility that they contrive with the untrusted cloud.

\section{REFERENCES}

[1]. M.Armbrust, A.Fox, R.Griffith, A.D.Joseph, R.Katz,A.Konwinski, G. Lee, D.Patterson, A.Rabkin, I.Stoica, andM.Zaharia. "A View of Cloud Computing,"Comm. ACM, vol. 53,no.4, pp.50-58, Apr.2010.

[2]. S.Kamara and K.Lauter,"Cryptographic Cloud Storage,” Proc.Int'l Conf. Financial Cryptography and Data Security (FC), pp.136-149, Jan. 2010.

[3]. M. Kallahalla, E. Riedel, R. Swaminathan, Q. Wang, and K.Fu,"Plutus: Scalable Secure File Sharing on Untrusted Storage," Proc.USENIX Conf. File and Storage Technologies, pp. 29-42, 2003.

[4]. E.Goh, H. Shacham, N. Modadugu, and D. Boneh, "Sirius: Securing Remote Untrusted Storage," Proc. Network and DistributedSystems Security Symp. (NDSS), pp. 131-145, 2003.
[5]. G. Ateniese, K. Fu, M. Green, and S. Hohenberger,"Improved Proxy Re-Encryption Schemes with Applications to Secure Distributed Storage," Proc. Network and Distributed Systems SecuritySymp. (NDSS), pp. 29-43, 2005.

[6]. Shucheng Yu, Cong Wang, Kui Ren, and Weijing Lou, "Achieving Secure, Scalable, and Fine-grained Data Access Control in Cloud Computing," Proc. ACM Symp. Information, Computer and Comm. Security, pp. 282-292, 2010.

[7]. V. Goyal, O. Pandey, A. Sahai, and B. Waters, "Attribute-Based Encryption for Fine-Grained Access Control of Encrypted Data," Proc. ACM Conf. Computer and Comm. Security (CCS), pp. 89-98, 2006

[8]. R. Lu, X. Lin, X. Liang, and X. Shen, "Secure Provenance: The Essential of Bread and Butter of Data Forensics in Cloud Computing," Proc. ACM Symp. Information, Computer and Comm. Security, pp. 282292, 2010.

[9]. B. Waters, "Ciphertext-Policy Attribute-Based Encryption: An Expressive, Efficient, and Provably Secure Realization," Proc. Int'l Conf. Practice and Theory in Public Key Cryptography Conf. Public Key Cryptography, http://eprint.iacr.org/2008/290.pdf, 2008

[10].Xuefeng Liu, Yuqing Zhang, Boyang Wang, and Jingbo Yang, "Mona: Secure Multi-Owner Data Sharing for Dynamic Groups in the Cloud," IEEE Transactions on Parallel and Distributed Systems, vol. 24, no. 6, pp. 1182-1191, June 2013.

[11].D.Boneh, X. Boyen, and E. Goh, "Hierarchical IdentityBasedEncryption with Constant Size Ciphertext," Proc. Ann. Int'l Conf.Theory and Applications of Cryptographic Techniques (EUROCRYPT),pp. 440-456, 2005. 
[12].C. Delerablee, P. Paillier, and D. Pointcheval, "FullyCollusionSecure Dynamic Broadcast Encryption with Constant-SizeCi-phertexts or Decryption Keys," Proc.First Int'l Conf. Pairing-BasedCryptography, pp. 39-59, 2007.

[13].Zhongma Zhu, Zemin Jiang, Rui Jiang, "The Attack on Mona: Secure Multi-Owner Data Sharing for Dynamic Groups in the Cloud,"Proceedings of2013 International Conference on Information Science and Cloud Computing (ISCC 2013), Guangzhou, Dec.7, 2013, pp. 185-189.

\section{BIOGRAPHIES}

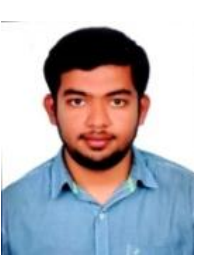

Mr. Kiran Kumar S Wali Completed BE electronics and communication in BLDEA's College Bijapaur, Under VTU Belgaum, Now pursuing M. tech Computer Networking in TJIT college Bangalore, under VTU Belgaum

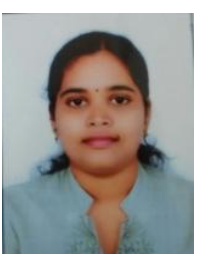

Ms. Bindhu. Madhavi:

Designation: Associate Professor in TJIT Bangalore. Experience 09+years of Teaching and Areas of Interest: Wireless Sensor Networks, AdHoc Networks and Image Processing.

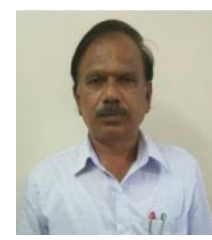

\section{Dr. M. Nagendra:}

Designation Associate Professor in S.K University ,Anantapur Karnataka 